\title{
SARS-CoV-2 infection and diabetes mellitus: A North Eastern Romanian experience
}

\author{
CARMEN MANCIUC $^{1}$, DRAGOS NEMESCU ${ }^{2}$, ANDREI VATA $^{1}$ and GEORGIANA ALEXANDRA LACATUSU ${ }^{3}$ \\ Departments of ${ }^{1}$ Infectious Diseases and ${ }^{2}$ Obstetrics and Gynecology, \\ University of Medicine and Pharmacy 'Grigore T. Popa', 700115 Iasi; \\ ${ }^{3}$ Department of Infectious Diseases, 'Sf. Parascheva' Clinical Hospital of Infectious Diseases, 700116 Iasi, Romania
}

Received September 28, 2020; Accepted October 26, 2020

DOI: $10.3892 /$ etm.2021.9710

\begin{abstract}
As it spread globally, the new SARS-CoV-2 virus was first confirmed in Romania in February 2020, inevitably infecting individuals with diabetes mellitus (DM) along the way. Diabetes is known to affect the response of the body to pathogens and, according to studies conducted in the last 3 months, it appears that diabetic patients are at a higher risk for developing severe forms of the disease and multiple complications. We performed a retrospective study in order to assess the patients with SARS-CoV-2 infection and DM admitted to 'Sf. Parascheva' Clinical Hospital of Infectious Diseases from March 4th until June 30th, 2020. Of the total 1,080 patients admitted during this period, 85 patients $(7.87 \%)$ had underlying DM, mostly type 2 (82 cases, $96.46 \%$ ); the mean age of these patients was 62 , and 42 were men (49.41\%). Chest CTs revealed indicative SARS-CoV-2 images for all patients and their treatment included individually tailored administration of hydroxychloroquine/lopinavir + ritonavir/enoxaparin sodium/tocilizumab/antibiotherapy according to the then national and international guidelines. In total, 70 patients $(82.35 \%)$ were cured and 15 succumbed to MODS and/or associated neoplasia, bringing the fatality rate to $17.64 \%$. Although advanced age and DM have been associated with aggravated forms of SARS-CoV-2 infection, over $80 \%$ of the patients included in the present study were cured. Nonetheless, diabetes appears to be a significant predictor of morbidity and mortality in the SARS-CoV-2 infection.
\end{abstract}

\section{Introduction}

In December 2019, a new strain of virus causing severe acute respiratory syndrome (SARS-CoV-2) appeared for the first

Correspondence to: Dr Dragos Nemescu, Department of Obstetrics and Gynecology, University of Medicine and Pharmacy 'Grigore T. Popa', Universitatii 16 Street, 700115 Iasi, Romania E-mail:dnemescu@yahoo.com

Key words: SARS-CoV-2, diabetes mellitus, intensive care unit, glycemic level, $\mathrm{C}$ reactive protein, fatality rate time in the city of Wuhan, Hubei province, China. The virus spread rapidly and, at the time of writing this study, more than 10 million cases were reported in 216 countries (1).

Coronaviruses $(\mathrm{CoV})$, generally known to cause acute respiratory infections, are enveloped viruses that have a single-stranded, positive-sense RNA genome (2). Although the majority of coronavirus infections affecting humans are mild, there have already been two important outbreaks of two types of coronaviruses causing multiple severe pneumonias: The severe acute respiratory syndrome coronavirus (SARS-CoV), with a mortality rate of up to $10 \%$, and Middle East respiratory syndrome coronavirus (MERS-CoV), with a mortality rate of over 34\% (3,4). Although SARS-CoV-2 has exhibited similar phylogenetic and clinical features with SARS-CoV, this new coronavirus appears to be considerably more easily transmissible, albeit less deadly (5). Typical symptoms, encountered in over $40 \%$ of the cases include fever, cough, fatigue and shortness of breath, and also less frequent common symptoms including anosmia and ageusia (6).

As the SARS-CoV-2 pandemic unfolded, diabetes mellitus (DM) was already one of the leading causes of morbidity and mortality throughout the world, and it is known that DM produces a plethora of macrovascular and microvascular complications that ultimately impact overall patient survival (7). In this new context, several studies reported apparent associations between diabetes mellitus, acute respiratory distress syndrome, more severe disease, and increased mortality (8-11). Multiple explanations have been put forth for this hypothesized association between DM as the underlying disease and the increased severity of SARS CoV-2 infection. Natural immunity, which is the primary line of defense against SARS-CoV-2, is inevitably weakened in patients that have uncontrolled DM; consequently, the virus proliferates unrestricted within the host (12). In fact, it has been demonstrated that even short-term hyperglycemia can briefly paralyze the natural immune system (13). In addition, DM promotes an increased pro-inflammatory cytokine response, mainly involving interleukin (IL)-1, IL-6 and tumor-necrosis factor (TNF)- $\alpha$, which further aggravates the prognosis of a SARS-CoV-2 infection (14).

The development and manufacturing of a vaccine against SARS-CoV-2 is an issue of utmost importance, however, it must be taken into consideration that it is going to take 
numerous months to produce a vaccine. Although several companies have announced that a vaccine against COVID-19 will be ready soon, this will be quite difficult to accomplish in reality considering that short- and long-term safety have to be taken into account (15).

\section{Materials and methods}

A retrospective observational study on confirmed SARS-CoV-2 patients admitted to 'Sf. Parascheva' Clinical Hospital of Infectious Diseases, Iasi, Romania was conducted between March 4th (the first such admission) and June 30th (the writing of the article). The primary inclusion criteria of the patients in the present study was represented by confirmed SARS-CoV-2 infection tested by RT-PCR assay which had as associated diagnosis type 1 or $2 \mathrm{DM}$. Patients that did not have a history of DM type 1 or 2 and/or had a negative result with RT-PCR testing were excluded from the present study.

The following data were collected: Demographic data, medical history, clinical and paraclinical data, blood tests, administered treatment and outcome. The RT-PCR tests were performed by either a molecular biology hospital laboratory or other accredited laboratories from Iasi county, Romania.

\section{Results}

From the beginning of the outbreak, a total of 1,080 patients were admitted to 'Sf. Parascheva' Clinical Hospital of Infectious Diseases of Iași, Romania. Of these patients, 85 (7.87\%) were known to be suffering from DM, mostly type 2 (82 cases, $96.46 \%$ ). Diabetic patients between $51-70$ years old were the most affected by the SARS-CoV-2 infection, the mean age being 62 (Fig. 1), and they presented in fairly equal proportions gender wise (42 were men, 49,41\%).

Other associated pathologies were cardiovascular (hypertension, heart failure, and atrial fibrillation), neurological (stroke, epilepsy), neoplastic, renal (kidney failure with or without chronic dialysis) (Fig. 2).

From the total of 1,080 patients admitted, 78 cases involving patients with associated underlying diseases, intensive care unit (ICU) therapy was required. Of these cases, 17 patients had DM as one of the underlying diseases. The majority of these patients (64 cases), presented with oxygen desaturation (Sp02<89\%), were dyspneic and polypneic, or they became hemodynamically unstable with their arterial pressure level dropping below $85 / 50 \mathrm{mmHg}$. Fifty-nine patients ultimately required orotracheal intubation and mechanical ventilation due to multiple complications including aspiration bronchopneumonia, sepsis, and multiple organ dysfunction syndrome (MODS).

In addition, in all cases, chest $\mathrm{C}$ Ts revealed lesions specific for SARS-CoV-2 infection, described either as ground-glass opacities or 'crazy paving' area patterns, linear densities or consolidations.

The treatment was administered according to the international and national guidelines available at the time and included hydroxychloroquine (HCQ)/lopinavir + ritonavir/enoxaparin sodium/tocilizumab/antibiotherapy. Each patient received individualized treatment based on the clinical and paraclinical data, as well as taking into consideration medical interactions.
During hospitalization, the management of associated cardiovascular, neurologic, neoplastic and renal comorbidities was ensured, alongside antibiotic treatment to address other types of infectious diseases whenever present, including urinary tract infections, sepsis, cellulitis, and Clostridium difficile infection. In addition, psychological therapy was performed upon request $(16,17)$.

Regarding the paraclinical data of patients with DM, glycemic levels over $200 \mathrm{mg} / \mathrm{dl}$ were observed in all the cases at the admittance and a median glycated hemoglobin level of $7.2 \%$ was calculated.

We took into consideration two groups of patients admitted to the ICU, group A, patients without DM (61 patients) and group B, patients with DM (17 patients). The fatality rate among the patients in group B was considerably higher $(88,23 \%, 15$ patients) than the rate of mortality among group B (59.01\%, 36 patients) (Fig. 3).

Regarding the glycemic levels of the patients with DM admitted to the ICU correlated with the level of $\mathrm{C}$ reactive protein (CRP), it was observed that CRP values $>70 \mathrm{mg} / \mathrm{l}$ were encountered in the patients that deceased. Patients admitted to the ICU with a CRP $<70 \mathrm{mg} / \mathrm{l}$ (patient 16 and 17) survived and were later transferred to the clinic (Fig. 4).

\section{Discussion}

The latest studies in scientific literature have been revealing a noteworthy association between increased mortality and morbidity in patients with SARS-CoV-2 and advanced age, severe obesity $\left(\mathrm{BMI} \geq 40 \mathrm{~kg} / \mathrm{m}^{2}\right)$, hypertension and $\mathrm{DM}$ as underlying diseases $(8,10,14,18,19)$. In the general population, the prevalence of DM is $8.5 \%$ (20) and among the admitted patients considered for the present study it was $7.87 \%$. In addition, the literature states that, elder patients are more affected by DM and often by other comorbidities, especially after the age of 60 (21-23), a fact that can also be sustained by the present study in which $>50 \%$ of the patients $(56.47 \%)$ were $>60$ years old. If we take into consideration that hypertension as well as cardiovascular disease are prevalent in DM patients, it is unclear whether DM independently contributes to this increased risk. However, diabetes has already been frequently reported to be associated with poor prognosis in other respiratory viral infections, mainly seasonal influenza, pandemic influenza A H1N1, SARS, and MERS $(24,25)$.

In March 2020, an Italian health institute reported 2,003 patients that succumbed from SARS-CoV-2 infection (26). Their median age was 80.5 , which was considerably higher than the median age 67.84 of the patients included in the present study. In addition, the same study stated that the prevalence of diabetes was $35.5 \%$ and that $70 \%$ of the total number of patients were men (26), while in our study gender differences were negligible ( $49.41 \%$ patients were men). Furthermore, in the largest case series reported by the Chinese Center for Disease Control and Prevention, performed on 72,314 cases of COVID-19, the patients with DM had a higher mortality (7.3\% in DM vs. $2.3 \%$ overall) (9).

Moutschen et al (27) and Knapp et al (28) acknowledge that poorly controlled diabetes inhibits lymphocyte proliferation, and also modifies the functions of neutrophils and 


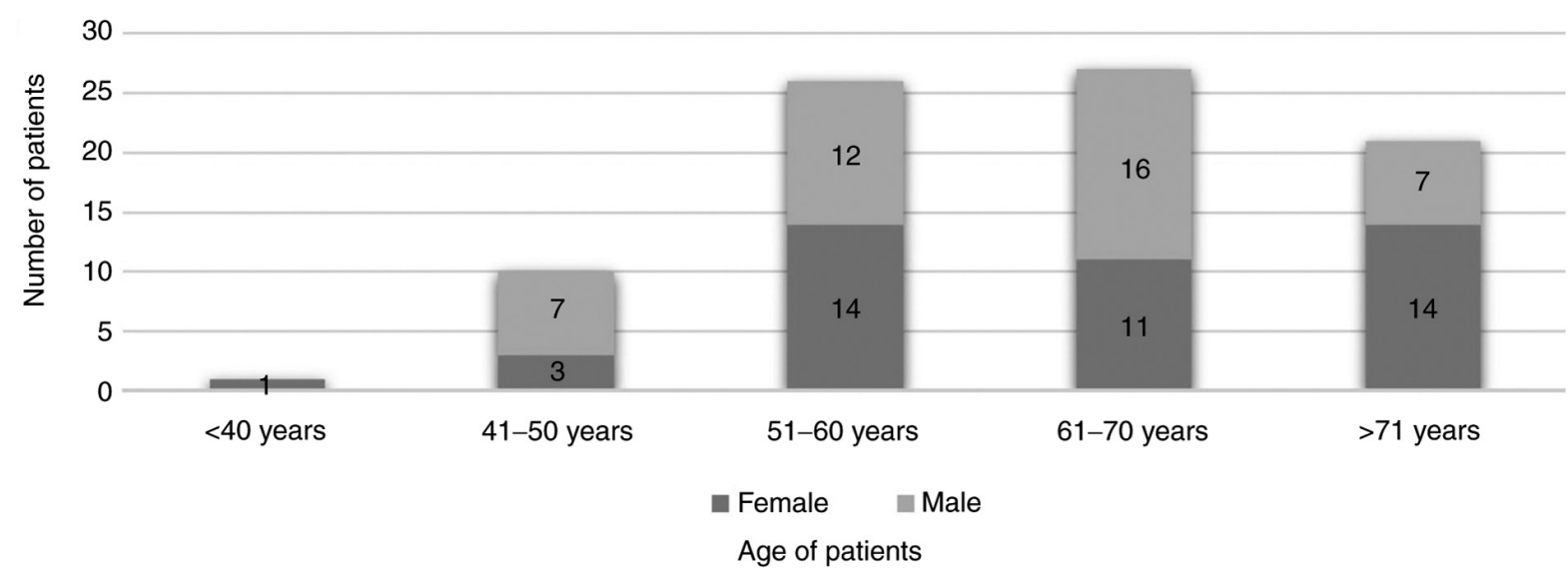

Figure 1. SARS-CoV-2 patient distribution by age and sex.

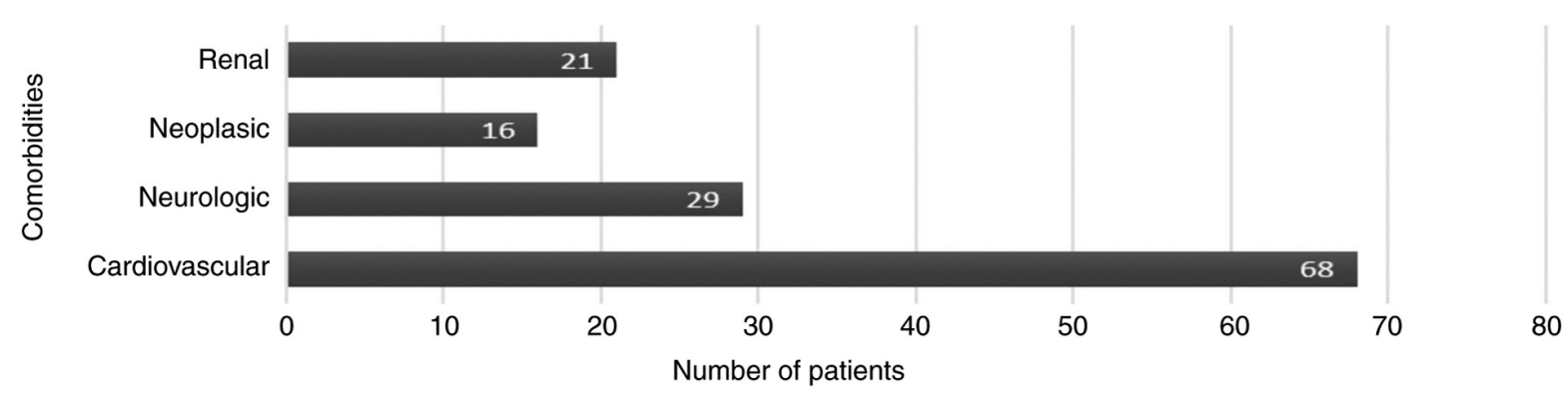

Figure 2. Other associated pathologies in SARS-CoV-2 DM patients. DM diabetes mellitus.

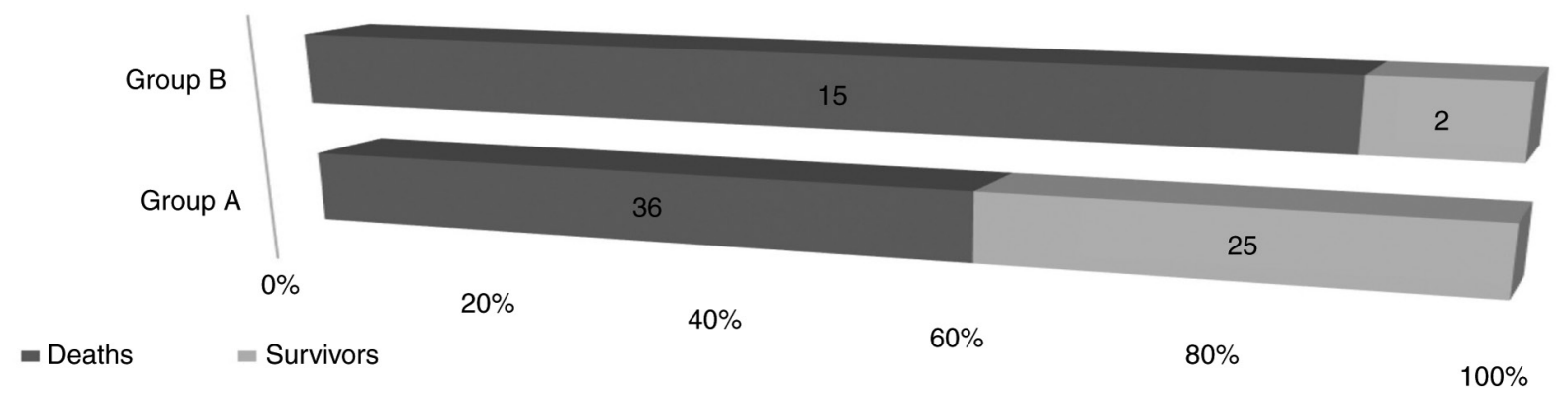

Figure 3. Distribution of the number of deaths and survivors with DM and without DM in SARS-CoV-2 patients admitted to the ICU. Group A, patients without DM; Group B, patients with DM. ICU, intensive care unit; DM diabetes mellitus.

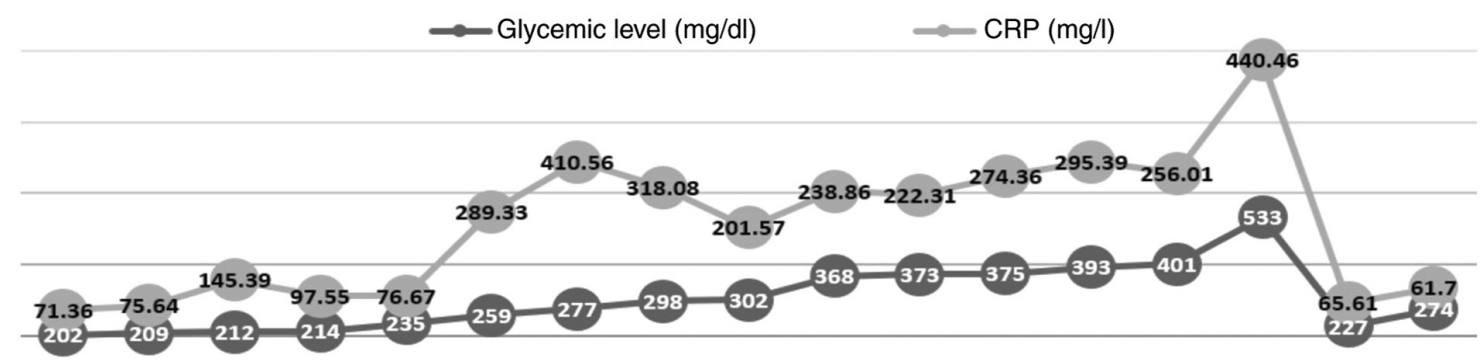

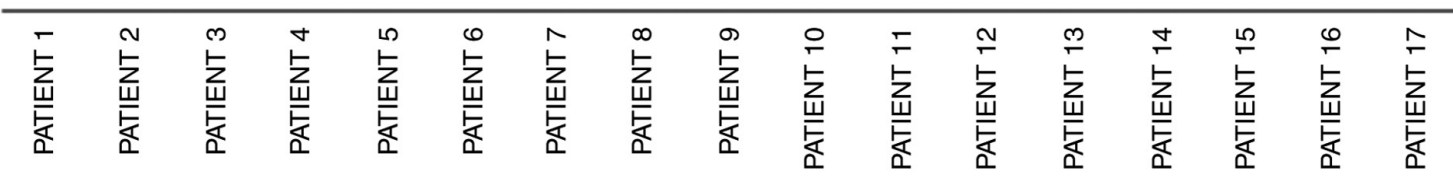

Figure 4. Association between the glycemic level and CRP in SARS-CoV-2 patients with DM admitted to the ICU. C reactive protein; DM diabetes mellitus; ICU, intensive care unit. 
monocytes/macrophages. Studies $(29,30)$ performed in vitro demonstrated that pulmonary epithelial cells respond to high glucose levels by significantly facilitating the replication of influenza virus. This indicates that hyperglycemia may contribute to increased viral replication in vivo (31), which can also apply to the case of the SARS-CoV-2 virus.

Furthermore, endothelial dysfunction and increased platelet aggregation have been associated with type $2 \mathrm{DM}$ and insulin resistance. These are flaws which support the development of a hypercoagulable pro-thrombotic state (32). Last but not least, patients with diabetes have also been revealed to have diminished forced vital capacity (FVC) and forced expiratory volume in one second (FEV1), which is associated with increased plasma glucose levels (33). This may explain the increased number of patients that required ICU therapy $(42.35 \%)$ and also the increased fatality rate of the patients with underlying DM.

Multiple studies (7,34-36) have determined the serum concentration of CRP in patients with COVID-19 and the results revealed that increased levels of CRP were observed in up to $86 \%$ of severe COVID-19 patients. CRP was found at increased levels in the severe group at the initial stage than those in the mild group (35) and also Luo et al observed in their study that patients who succumbed to COVID-19 had approximately 10 -fold higher levels of CRP than the recovered patients (37). In the present study, the patients admitted to the ICU that succumbed had an increase of CRP value between 14-88-fold (71.36-440.46 mg/l).

In conclusion, uncontrolled DM appears to be a significant predictor of mortality, not only because of how it modifies physiological mechanisms but also by how it predisposes the patient to multiple complications. However, even in the presence of advanced age and DM, $82.35 \%$ from the total number of the patients included in the study were cured. Careful assessment of the numerous components that contribute to poor prognosis of the patients with diabetes infected with SARS-CoV-2 virus may represent the best way to overcome the current situation.

\section{Acknowledgements}

Not applicable.

\section{Funding}

No funding was received.

\section{Availability of data and materials}

All data generated or analyzed during this study are included in this published article and its supplementary files.

\section{Authors' contributions}

$\mathrm{CM}$ and GAL designed the study. DN and AV contributed to data extraction and quality assessment. CM, DN and GAL were responsible for the analysis and discussion of the data. $\mathrm{CM}$ and GAL drafted the manuscript. AV and DN critically revised the manuscript for important intellectual content. All authors read and approved the final manuscript.

\section{Ethics approval and consent to participate}

Not applicable.

\section{Patient consent for publication}

Not applicable.

\section{Competing interests}

The authors declare that they have no competing interests.

\section{References}

1. Coronavirus disease (COVID-19) Weekly Epidemiological Update and Weekly Operational Update. https://www.who.int/docs/defaultsource/coronaviruse/20200630-covid-19-sitrep-162.pdf?sfvrsn= e00a5466_2. Accessed June 30, 2020.

2. Cui J, Li $\bar{F}$ and Shi ZL: Origin and evolution of pathogenic coronaviruses. Nat Rev Microbiol 17: 181-192, 2019.

3. Song Z, Xu Y, Bao L, Zhang L, Yu P, Qu Y, Zhu H, Zhao W, Han Y and Qin C: From SARS to MERS, thrusting coronaviruses into the spotlight. Viruses 11: 59, 2019.

4. Docea AO, Tsatsakis A, Albulescu D, Cristea O, Zlatian O, Vinceti M, Moschos SA, Tsoukalas D, Goumenou M, Drakoulis N, et al: A new threat from an old enemy: Re-emergence of coronavirus (Review). Int J Mol Med 45: 1631-1643, 2020.

5. Ceccarelli M, Berretta M, Venanzi Rullo E, Nunnari G and Cacopardo B: Differences and similarities between Severe Acute Respiratory Syndrome (SARS)-CoronaVirus $(\mathrm{CoV})$ and SARS-CoV-2. Would a rose by another name smell as sweet? Eur Rev Med Pharmacol Sci 24: 2781-2783, 2020.

6. Tanasa IA, Manciuc C, Carauleanu A, Navolan DB, Bohiltea RE and Nemescu D: Anosmia and ageusia associated with coronavirus infection (COVID-19)-what is known? Exp Ther Med 20: 2344-2347, 2020.

7. Williams R, Karuranga S, Malanda B, Saeedi P, Basit A, Besançon S, Bommer C, Esteghamati A, Ogurtsova K, Zhang P and Colagiuri S: Global and regional estimates and projections of diabetes-related health expenditure: Results from the International Diabetes Federation Diabetes Atlas, 9th edition. Diabetes Res Clin Pract 162: 108072, 2020.

8. Guan WJ, Ni ZY, Hu Y, Liang WH, Ou CQ, He JX, Liu L, Shan H, Lei CL, Hui DSC, et al: China medical treatment expert group for Covid-19: Clinical characteristics of coronavirus disease 2019 in China. N Engl J Med 382: 1708-1720, 2020.

9. Wu Z and McGoogan JM: Characteristics of and important lessons from the coronavirus disease 2019 (COVID-19) Outbreak in China: Summary of a Report of 72314 cases from the Chinese center for disease control and prevention. JAMA 323: 1239-1242, 2020.

10. Yang X, Yu Y, Xu J, Shu H, Xia J, Liu H, Wu Y, Zhang L, Yu Z, Fang M, et al: Clinical course and outcomes of critically ill patients with SARS-CoV-2 pneumonia in Wuhan, China: A single-centered, retrospective, observational study. Lancet Respir Med 8: 475-481, 2020.

11. Wu C, Chen X, Cai Y, Xia J, Zhou X, Xu S, Huang H, Zhang L, Zhou X, Du C, et al: Risk factors associated with acute respiratory distress syndrome and death in patients with coronavirus disease 2019 pneumonia in Wuhan, China. JAMA Intern Med 180: 934-943, 2020.

12. Geerlings SE and Hoepelman AI: Immune dysfunction in patients with diabetes mellitus (DM). FEMS Immunol Med Microbiol 26: 259-265, 1999.

13. Jafar N, Edriss H and Nugent K: The effect of short-term hyperglycemia on the innate immune system. Am J Med Sci 351: 201-211, 2016.

14. Onder G, Rezza G and Brusaferro S: Case-fatality rate and characteristics of patients dying in relation to COVID-19 in Italy. JAMA 323: 1775-1776, 2020.

15. Calina D, Docea AO, Petrakis D, Egorov AM, Ishmukhametov AA, Gabibov AG, Shtilman MI,Kostoff R, Carvalho F, Vinceti M, et al: Towards effective COVID-19 vaccines: Updates, perspectives and challenges (Review). Int J Mol Med 46: 3-16, 2020.

16. Manciuc $C$ and Largu A: Impact and risk of institutionalized environments on the psyho-emotional development of the HIV-positive youth. Environ Eng Manag J 13: 3123-3129, 2014. 
17. Manciuc C, Filip-Ciubotaru F, Badescu A, Duceag LD and Largu AM: The patient-doctor-psychologist triangle in a case of severe imunosupression in the HIV infection. Rev Med Chir Soc Med Nat Iasi 120: 119-123, 2016.

18. Centers for Disease Control and Prevention: National Diabetes Statistics Report, 2020. Centers for Disease Control and Prevention, US Department of Health and Human Services, Atlanta, GA, pp9, 2020.

19. Yang J, Zheng Y, Gou X, Pu K, Chen Z, Guo Q, Ji R, Wang H, Wang Y and Zhou Y: Prevalence of comorbidities and its effects in patients infected with SARS-CoV-2: A systematic review and meta-analysis. Int J Infect Dis 94: 91-95, 2020.

20. Emerging Risk Factors Collaboration; Sarwar N, Gao P, Seshasai SR, Gobin R, Kaptoge S, Di Angelantonio E, Ingelsson E, Lawlor DA, Selvin E, et al: Diabetes mellitus, fasting blood glucose concentration, and risk of vascular disease: A collaborative meta-analysis of 102 prospective studies. Lancet 375: 2215-2222, 2010.

21. Chentli F, Azzoug S and Mahgoun S: Diabetes mellitus in elderly. Indian J Endocrinol Metab 19: 744-752, 2015.

22. Davis JW, Chung R and Juarez DT: Prevalence of comorbid conditions with aging among patients with diabetes and cardiovascular disease. Hawaii Med J 70: 209-213, 2011.

23. Tyrovolas S, Koyanagi A, Garin N, Olaya B, Ayuso-Mateos JL, Miret M, Chatterji S, Tobiasz-Adamczyk B, Koskinen S, Leonardi M, et al: Diabetes mellitus and its association with central obesity and disability among older adults: A global perspective. Exp Gerontol 64: 70-77, 2015.

24. Hong KW, Cheong HJ, Choi WS, Lee J, Wie SH, Baek JH, Kim HY, Jeong HW and Kim WJ: Clinical courses and outcomes of hospitalized adult patients with seasonal influenza in Korea, 2011-2012: Hospital-based Influenza Morbidity \& Mortality (HIMM) surveillance. J Infect Chemother 20: 9-14, 2014.

25. Schoen K, Horvat N, Guerreiro NFC, de Castro I and de Giassi KS: Spectrum of clinical and radiographic findings in patients with diagnosis of $\mathrm{H} 1 \mathrm{~N} 1$ and correlation with clinical severity. BMC Infect Dis 19: 964, 2019.

26. Istituto Superiore di Sanita: Report of characteristics of patients died positive for COVID-19 in Italy. Accesed May 21, 2020.
27. Moutschen MP, Scheen AJ and Lefebvre PJ: Impaired immune responses in diabetes mellitus: Analysis of the factors and mechanisms involved. Relevance to the increased susceptibility of diabetic patients to specific infections. Diabete Metab 18: 187-201, 1992.

28. Knapp S: Diabetes and infection: Is there a link?-A mini-review. Gerontology 59: 99-104, 2013.

29. Reading PC, Allison J, Crouch EC and Anders EM: Increased susceptibility of diabetic mice to influenza virus infection: Compromise of collectin-mediated host defense of the lung by glucose? J Virol 72: 6884-6887, 1998.

30. Ilyas R, Wallis R, Soilleux EJ, Townsend P, Zehnder D, Tan BK, Sim RB, Lehnert H, Randeva HS and Mitchell DA: High glucose disrupts oligosaccharide recognition function via competitive inhibition: A potential mechanism for immune dysregulation in diabetes mellitus. Immunobiology 216: 126-131, 2011.

31. Kohio HP and Adamson AL: Glycolytic control of vacuolar-type ATPase activity: A mechanism to regulate influenza viral infection. Virology 444: 301-309, 2013.

32. Dunn EJ and Grant PJ: Type 2 diabetes: An atherothrombotic syndrome. Curr Mol Med 5: 323-332, 2005

33. Lange P, Groth S, Kastrup J, Mortensen J, Appleyard M, Nyboe J, Jensen G and Schnohr P: Diabetes mellitus, plasma glucose and lung function in a cross-sectional population study. Eur Respir J 2: 14-19, 1989.

34. Chen N, Zhou M, Dong X, Qu J, Gong F, Han Y, Qiu Y, Wang J, Liu Y, Wei Y, et al: Epidemiological and clinical characteristics of 99 cases of 2019 novel coronavirus pneumonia in Wuhan, China: A descriptive study. Lancet 395: 507-513, 2020.

35. Chen T, Wu D, Chen H, Yan W, Yang D, Chen G, Ma K, Xu D, Yu H, Wang H, et al: Clinical characteristics of 113 deceased patients with coronavirus disease 2019: Retrospective study. BMJ 368: m1091, 2020.

36. Gao Y, Li T, Han M, Li X, Wu D, Xu Y, Zhu Y, Liu Y, Wang X and Wang L: Diagnostic utility of clinical laboratory data determinations for patients with the severe COVID-19. J Med Virol 92: 791-796, 2020.

37. Luo X, Zhou W, Yan X, Guo T, Wang B, Xia H, Ye L, Xiong J, Jiang Z, Liu Y, et al: Prognostic value of C-reactive protein in patients with COVID-19. Clin Infect Dis: May 23, 2020 (Epub ahead of print). doi: 10.1093/cid/ciaa641. 